\title{
Estudio y caracterización del efecto de compuestos con titanio sobre el sistema hidruro Li-B-Mg-H con alta capacidad de almacenamiento de hidrógeno
}

\author{
Hernán Martinelli ${ }^{1}$, Victoria Castro Riglos ${ }^{2,4}$, \\ Julián Puszkiel ${ }^{3,4}$
}

\author{
${ }^{1}$ Departamento de Física, Facultad de Ciencias Exactas y Naturales, Universidad de Buenos Aires, CABA, Buenos Aires, \\ Argentina. e-mail: hmartinelli1@ @otmail.com \\ ${ }^{2}$ División Física de Metales \& ${ }^{3}$ Departamento de Físico-Química de Materiales, Centro Atómico Bariloche (CAB), Av. \\ Bustillo 9500, Bariloche, Río Negro, Argentina. \\ ${ }^{4}$ CONICET, Buenos Aires, Argentina. \\ e-mail: viquiriglos@gmail.com; julianpuszkiel1979@gmail.com
}

\section{RESUMEN}

La falta de un medio para el almacenamiento de hidrógeno de modo seguro y eficiente es uno de los principales inconvenientes para el empleo del hidrógeno como nuevo vector de energía. El almacenamiento de hidrógeno en estado sólido mediante la formación de compuestos hidruros presenta la ventaja de alcanzar altas capacidades de almacenamiento a temperaturas y presiones moderadas.

En el presente trabajo se investiga el efecto de aditivos en base titanio $\left(\mathrm{TiB}_{2}, \mathrm{Li}_{\mathrm{x}} \mathrm{TiO}_{2}\right)$ sobre el comportamiento cinético del sistema hidruro reactivo $2 \mathrm{LiBH}_{4}+\mathrm{MgH}_{2}$. Los resultados obtenidos muestran que la presencia de partículas nanométricas de fases titanato $\left(\mathrm{Li}_{\mathrm{x}} \mathrm{TiO}_{2}\right)$ mejoran notablemente la primera deshidrogenación no isotérmica del $2 \mathrm{LiBH}_{4}+\mathrm{MgH}_{2}$, reduciendo el tiempo de liberación completa de hidrógeno desde $35 \mathrm{~h}$. hasta $2 \mathrm{~h}$. A su vez, la adición de $\mathrm{Li}_{\mathrm{x}} \mathrm{TiO}_{2}$ mejora las cinéticas de hidrogenación/deshidrogenación isotérmicas. Mediante el empleo de técnicas avanzadas como la microscopía electrónica de transmisión y la espectroscopía Raman, entre otras, se evidenció que adición de $\mathrm{Li}_{\mathrm{x}} \mathrm{TiO}_{2}$ al sistema hidruro $2 \mathrm{LiBH}_{4}+\mathrm{MgH}_{2}$ permite la absorción/desorción de hidrógeno través de un mecanismo novedoso y reversible.

Palabras clave: Hidrógeno, Composites hidruros reactivos, Dióxido de titanio, Cinética de titanatos.

\begin{abstract}
One of the main constraints for the implementation of hydrogen as energy carrier is the lack of an efficient and safe hydrogen storage system. Hydrogen storage in solid state through hydride compounds formation is a potential alternative to address this problem.

In this work, it is studied the effect of different titanium based compounds $\left(\mathrm{TiB}_{2}, \mathrm{Li}_{\mathrm{x}} \mathrm{TiO}_{2}\right)$ on the kinetic behavior of $2 \mathrm{LiBH}_{4}+\mathrm{MgH}_{2}$ reactive hydride composite. It is found that nanometric particles of titanates $\left(\mathrm{Li}_{\mathrm{x}} \mathrm{TiO}_{2}\right)$ noticeably improve the first non-isothermal dehydrogenation behavior of the $2 \mathrm{LiBH}_{4}+\mathrm{MgH}_{2}$, reducing the complete $\mathrm{H}_{2}$ release time from $35 \mathrm{~h}$ to $2 \mathrm{~h}$. Moreover, the $\mathrm{Li}_{\mathrm{x}} \mathrm{TiO}_{2}$ addition improves the isothermal hydrogenation/dehydrogenation kinetic behavior. The information gained by advanced techniques such as transmission electron microscopy and Raman spectroscopy, among others, help us to elucidate that the addition of $\mathrm{Li}_{\mathrm{x}} \mathrm{TiO}_{2}$ to $2 \mathrm{LiBH}_{4}+\mathrm{MgH}_{2}$ allows the hydrogen uptake/release via a novel and reversible mechanism.
\end{abstract}

Keywords: Hydrogen, Reactive hydride composites, Titanium dioxide, Titanates, Kinetics.

\section{INTRODUCCIÓN}

Una de las alternativas de energías limpias más prometedoras que ha surgido en este último tiempo es el desarrollo de tecnologías a base de hidrógeno, el cual se encuentra en abundancia combinado con el oxigeno en el agua. A pesar de tener un gran potencial como vector energético, la implementación del hidrógeno presenta inconvenientes tecnológicos no resueltos, entre ellos el almacenamiento del mismo de modo eficiente y seguro para aplicaciones estacionarias y móviles. El almacenamiento en estado sólido mediante la formación 
de compuestos hidruros se ha presentado como una potencial solución a este problema. La formación de compuestos hidruros permite alcanzar altas capacidades de almacenamiento de hidrógeno a temperaturas y presiones menos severas que las requeridas para almacenamiento de hidrógeno en estado líquido o gaseoso [1]. El sistema hidruro compuesto por $2 \mathrm{LiH}+\mathrm{MgB}_{2} / 2 \mathrm{LiBH}_{4}+\mathrm{MgH}_{2}$ se presenta como un prometedor candidato para almacenamiento de hidrógeno dadas su alta capacidad gravimétrica teórica $\left(11,4 \% \mathrm{p} / \mathrm{p} \mathrm{H}_{2}\right)$ y su propicia estabilidad termodinámica $\left(40,5 \mathrm{~kJ} / \mathrm{mol} \mathrm{H}\right.$ ) de acuerdo con la reacción: $2 \mathrm{LiBH}_{4(\mathrm{l})}+\mathrm{MgH}_{2(\mathrm{~s})} \leftrightarrows$ $2 \mathrm{LiH}_{(\mathrm{s})}+\mathrm{MgB}_{2(\mathrm{~s})}+4 \mathrm{H}_{2(\mathrm{~g})}$ [2]. No obstante, el proceso de liberación de hidrógeno en condiciones dinámicas ocurre en dos etapas como lo describe la siguiente reacción: $2 \mathrm{LiBH}_{4(\mathrm{l})}+\mathrm{MgH}_{2(\mathrm{~s})} \rightarrow \mathrm{Mg}_{(\mathrm{s})}+2 \mathrm{LiBH}_{4(\mathrm{l})}+\mathrm{H}_{2(\mathrm{~g})}$ $\rightarrow 2 \mathrm{LiH}_{(\mathrm{s})}+\mathrm{MgB}_{2(\mathrm{~s})}+4 \mathrm{H}_{2(\mathrm{~g})}$ y toma largos tiempos a relativamente altas temperaturas de trabajo. A su vez, el proceso de deshidrogenación requiere de cierta sobrepresión de hidrógeno para posibilitar la formación de la fase $\mathrm{MgB}_{2}$, responsable de la absorción-desorción reversible de hidrógeno [3,4]. Previamente se ha demostrado que el agregado de $1 \%$ mol de $\mathrm{TiO}_{2}$ mejora significativamente la cinética de deshidruración con altas capacidades de almacenamiento $\left(9,5 \% \mathrm{p} / \mathrm{p} \mathrm{H}_{2}\right)$ y notable estabilidad al ciclado dada la formación in-situ de partículas nanométricas de fases con titanio: $\mathrm{TiB}_{2}$ y principalmente $\mathrm{Li}_{\mathrm{x}} \mathrm{TiO}_{2}$ [5]. Sin embargo, no se ha clarificado aún cual es el mecanismo por el cual dichas nanopartículas mejoran notablemente las propiedades cinéticas del sistema hidruro $2 \mathrm{LiH}+\mathrm{MgB}_{2} / 2 \mathrm{LiBH}_{4}+\mathrm{MgH}_{2}$. Por lo tanto, en el presente trabajo se investiga el efecto y mecanismo del agregado de $5 \%$ mol $\mathrm{TiB}_{2} \mathrm{y} \mathrm{Li}_{\mathrm{x}} \mathrm{TiO}_{2}(\mathrm{x}=0,07-0,5)$ sobre el comportamiento cinético de deshidrogenación-hidrogenación de la mezcla estequiométrica $2 \mathrm{LiBH}_{4}+\mathrm{MgH}_{2}$.

\section{MATERIALES Y MÉTODOS}

Mezclas de polvos estequiométricas de $2 \mathrm{LiBH}_{4}+\mathrm{MgH}_{2}$ sin y con el agregado de $5 \%$ en mol de $\mathrm{TiB}_{2}$ y $\mathrm{Li}_{\mathrm{x}} \mathrm{TiO}_{2}$ $(\mathrm{x}=0,07$ y 0,5$)$ se prepararon por molienda mecánica (MM). El proceso de molienda mecánica se llevó a cabo en un molino Fritsch P6-Planetary empleando una cámara hermética de $80 \mathrm{~cm}^{3}$, bolas de acero inoxidable como medio de molienda con una relación bola a polvo de 40:1 y una velocidad de disco de $400 \mathrm{rpm}$. El tiempo de molienda fue de $2 \mathrm{~h}$ y la atmósfera de molienda fue argón. Los materiales de partida utilizados fueron: $\mathrm{LiBH}_{4}$ (Sigma Aldritch, polvo, pureza >90\%), $\mathrm{MgH}_{2}$ (Alfa Aesar, polvo premolido $10 \mathrm{~h}$, pureza $98 \%$ ), $\mathrm{TiO}_{2}(\mathrm{Sigma}$ Aldrich, 99,99\%), $\mathrm{TiB}_{2}$ (Sigma Aldritch, polvo premolido $5 \mathrm{~h}$ ). El $\mathrm{Li}_{\mathrm{x}} \mathrm{TiO}_{2}($ mezcla de $\mathrm{x}=0,07$ y 0,50$)$ se preparó a partir de una mezcla estequiométrica $0,5 \mathrm{LiH}+\mathrm{TiO}_{2}$ por medio de la molienda mecánica durante $2 \mathrm{~h}$. A lo largo del trabajo los materiales compuestos por $2 \mathrm{LiBH}_{4}+\mathrm{MgH}_{2}, \quad 2 \mathrm{LiBH}_{4}+\mathrm{MgH}_{2}+5 \mathrm{~mol}_{2} \mathrm{TiB}_{2}$, y $2 \mathrm{LiBH}_{4}+\mathrm{MgH}_{2}+5 \mathrm{~mol} \% \mathrm{Li}_{\mathrm{x}} \mathrm{TiO}_{2}$ se designan: $2 \mathrm{LBHMH}, 2 \mathrm{LBHMH} 5 \mathrm{TiB}_{2}$ y $2 \mathrm{LBHMH} \mathrm{Li}_{\mathrm{x}} \mathrm{TiO}_{2}$.

Las propiedades cinéticas del material se evaluaron en un equipo tipo Sieverts modificado con controladores de flujo. Las mediciones de deshidruración e hidruración se llevaron a cabo sometiendo los materiales a una rampa de temperatura de $10^{\circ} \mathrm{C} /$ min desde $\sim 30{ }^{\circ} \mathrm{C}$ hasta $425{ }^{\circ} \mathrm{C}$ y con una contrapresión de hidrógeno de 3 bar y a temperatura constante de $425{ }^{\circ} \mathrm{C}$ y $400{ }^{\circ} \mathrm{C}$ a 3 bar y 50 bar de hidrógeno, respectivamente. La masa de material empleada fue de entre 120-150 mg. Luego de la MM y la interacción con hidrógeno se caracterizaron los materiales mediante distintas técnicas: difracción de rayos X (DRX), calorimetría diferencial de barrido de alta presión (CDB-AP) y termogravimetría (TG). A su vez, los materiales deshidrurados se caracterizaron por DRX, microscopía electrónica de transmisión (MET) y espectroscopía Raman (ER). La caracterización por DRX se realizó en un equipo PANanalytical Empyrean (radiación CuKa, $\lambda$ $=1.5405 \AA$ Å $30 \mathrm{~mA}$ y $40 \mathrm{kV}$ ) mediante el empleo de una cámara especialmente diseñada para mantener una atmósfera inerte durante la medición. El tamaño de grano del $\mathrm{MgB}_{2}$ (101) se determinó por el método de Scherrer [6]. Las curvas de CDB-AP se midieron en un equipo TA Instruments 2910 a una velocidad de calentamiento de $5^{\circ} \mathrm{C} / \mathrm{min}$ y en una atmósfera de 3 bar de hidrógeno. Las mediciones de TG se llevaron a cabo en una balanza TGA-HP50F - TA Instruments a una velocidad de calentamiento de $5^{\circ} \mathrm{C} / \mathrm{min}$ y en flujo de hidrógeno de $50 \mathrm{~cm}^{3} / \mathrm{min}$, manteniendo la sobrepresión en 3 bar de hidrógeno. Las observaciones por MET se realizaron en un microscopio Tecnai $\mathrm{G}^{2}$ de resolución espacial de $0,12 \mathrm{~nm}$ y con cañón de emisión de campo. La tensión de aceleración empleada fue de $200 \mathrm{kV}$. Las muestras en estado sólido en forma de polvo fueron dispersadas en hexano en un baño ultrasónico durante 10 minutos y luego depositadas en una grilla de cobre con sustrato de carbono amorfo tipo Formvar para su posterior análisis por MET. Los materiales fueron observados empleando muestras del mismo lote de preparación y se realizaron 5 observaciones para cada material, de las cuales se obtuvieron aproximadamente 50 fotos que fueron analizadas para poder confirmar las conclusiones. Para el análisis de los resultados obtenidos por MET se utilizaron los siguientes programas: Digital Micrograph (licencia $\mathrm{N}^{\circ}$ 90294175), i-TEM (licencia $\mathrm{N}^{\circ}$ A2382500) y JEMs (licencia $\mathrm{N}^{\circ}$ IEb59yBDflUMh). Los espectros Raman se obtuvieron utilizando un microscopio Raman Confocal Jobin Yvon MicroRaman T64000 NIR-UV con un objetivo de x20, a una longitud de onda de $514 \mathrm{~nm}$ y una potencia de $15 \mathrm{~mW}$. Las muestras fueron encapsuladas en porta-muestras herméticos para poder realizar las mediciones sin que el material se deteriore.

En algunas instancias particulares, como por ejemplo luego del proceso de MM y tratamientos 
térmicos, la presencia de hidrógeno se verificó mediante el empleo de un detector de gases combustibles Bacharach. Para ello y con el fin de no dañar el material, se utilizaron tuberías provistas de válvulas y conectadas a bombas de vacío especialmente construidas para poder tomar una muestra de la fase gaseosa y luego exponer el gas al mencionado detector.

Los materiales de partida, los obtenidos de la preparación y luego de la interacción con hidrógeno fueron manipulados en caja de guantes MBraun Unilab con concentración de oxígeno y humedad <5 ppm.

\section{RESULTADOS Y DISCUSIÓN}

La presente sección se divide en tres subsecciones. Primero se muestran y analizan los resultados obtenidos del comportamiento cinético de los materiales formadores de hidruros. Luego se interpreta dicho comportamiento mediante la caracterización de los tipos de fases cristalinas y amorfas presentes, distribución de tamaños de los aditivos y el análisis térmico de los materiales luego de la preparación y en distintas etapas de la interacción con hidrógeno. Finalmente, se propone un mecanismo de acción del aditivo en base titanio que mostró un efecto beneficioso sobre las propiedades cinéticas del sistema hidruro $2 \mathrm{LiBH}_{4}+\mathrm{MgH}_{2}$.

\subsection{Comportamiento cinético deshidrogenación y reversibilidad al ciclado en hidrógeno}

La figura 1 muestra el comportamiento cinético de la primera desorción no-isotérmica de hidrógeno hasta $425^{\circ} \mathrm{C}$ para el material $2 \mathrm{LBHMH}$ sin y con aditivos $\left(\mathrm{TiB}_{2}, \mathrm{Li}_{\mathrm{x}} \mathrm{TiO}_{2}\right)$.

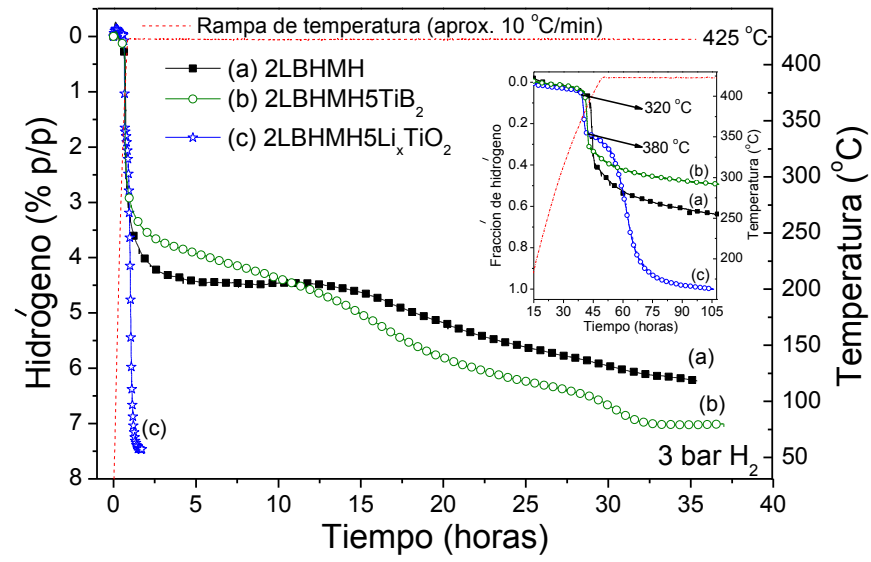

Figura 1: Comportamiento cinético de la primera desorción no-isotérmica hasta $425^{\circ} \mathrm{C}$ y 3 bar de $\mathrm{H}_{2}$ para (a) $2 \mathrm{LBHMH}$, (b) $2 \mathrm{LBHMH}_{5} \mathrm{TiB}_{2}$ and (c) $2 \mathrm{LBHMH} 5 \mathrm{Li}_{\mathrm{x}} \mathrm{TiO}_{2}$.

Se puede apreciar claramente que el proceso de deshidrogenación ocurre en tres etapas para material 2LBHMH (fig. 1 (a)): una primera etapa muy rápida que corresponde a la descomposición de $\mathrm{MgH}_{2}$ (reacción 1), una segunda etapa en la que la liberación de hidrógeno es mínima y se encuentra relacionado con la formación del compuesto $\mathrm{Li}_{2} \mathrm{~B}_{12} \mathrm{H}_{12}$ amorfo y muy estable (reacción 2). La presencia de $\mathrm{Li}_{2} \mathrm{~B}_{12} \mathrm{H}_{12}$ no permite un contacto eficiente entre el $\mathrm{Mg}$ formado en la primera etapa y el $\mathrm{LiBH}_{4}$ para la obtención de $\mathrm{MgB}_{2}$ [4]. Finalmente, una tercera etapa lenta donde se libera hidrógeno y forma $\mathrm{MgB}_{2}$ e $\mathrm{LiH}$ (reacción 3) [7]. Si bien el porcentaje gravimétrico teórico de hidrógeno almacenado en el material 2LBHMH es de 11,4\% p/p, se puede observar que se ha obtenido sólo un $6 \%$ p/p de hidrógeno luego de 35 h (fig. 1 (a)). Esto indica que el proceso de deshidrogenación es incompleto.

Primera etapa

$$
2 \mathrm{LiBH}_{4(\mathrm{l})}+\mathrm{MgH}_{2(\mathrm{~s})} \rightarrow \mathrm{Mg}_{(\mathrm{s})}+2 \mathrm{LiBH}_{4(\mathrm{l})}+\mathrm{H}_{2(\mathrm{~g})}
$$

Segunda etapa

$$
2 \mathrm{LiBH}_{4(\mathrm{l})} \rightarrow(1 / 6) \mathrm{Li}_{2} \mathrm{~B}_{12} \mathrm{H}_{12(\mathrm{~s})}+(5 / 3) \mathrm{LiH}_{(\mathrm{s})}+(13 / 6) \mathrm{H}_{2(\mathrm{~g})}
$$

Tercera etapa

$$
\mathrm{LiBH}_{4(\mathrm{l})}+\mathrm{Mg}_{(\mathrm{s})} \rightarrow 2 \mathrm{LiH}_{(\mathrm{s})}+\mathrm{MgB}_{2(\mathrm{~s})}+3 \mathrm{H}_{2(\mathrm{~g})}
$$

El tiempo requerido para la primera deshidruración se ve poco reducido mediante el agregado de $\mathrm{TiB}_{2}$ (fig. 1 (b)). Sin embargo, es de notar que la reacción pareciera ocurrir en dos etapas, siendo la segunda marcadamente lenta. Bösenberrg et al. [8] han propuesto que la presencia de boruros conformados de metales 
de transición como $\mathrm{TiB}_{2}, \mathrm{NbB}_{2}$, etc., con igual estructura cristalina hexagonal que el $\mathrm{MgB}_{2}$ mejoran las propiedades cinéticas de deshidrogenación del sistema hidruro $2 \mathrm{LiH}+\mathrm{MgB}_{2} / 2 \mathrm{LiBH}_{4}+\mathrm{MgH}_{2}$, dado que proveen un medio para la nucleación y crecimiento rápida de la fase $\mathrm{MgB}_{2}$. No obstante, el efecto observado mediante la presencia de $\mathrm{TiB}_{2}$ no es tan marcado como el que se observa con el aditivo $\mathrm{Li}_{\mathrm{x}} \mathrm{TiO}_{2}$. Durante la primera deshidruración (fig. 1 (d)) el agregado de $\mathrm{Li}_{\mathrm{x}} \mathrm{TiO}_{2}$ elimina la segunda etapa (reacción 2) y reduce la liberación completa de hidrógeno a menos de $2 \mathrm{~h}$ (capacidad de entre $7-8 \% \mathrm{p} / \mathrm{p}$ de $\mathrm{H}_{2}$ cercana a la teórica teniendo en cuenta la presencia del aditivo). Por lo tanto la primera deshidrogenación del material $2 \mathrm{LBHMH}_{5} \mathrm{Li}_{\mathrm{x}} \mathrm{TiO}_{2}$ procede según la reacción (4). Adicionalmente, las temperaturas para el comienzo de la descomposición del $\mathrm{MgH}_{2}$ y $\mathrm{LiBH}_{4}$ se ven reducidas en $\sim 50{ }^{\circ} \mathrm{C}$ respecto del material sin aditivo (fig. 1 , gráfico inserto).

$$
2 \mathrm{LiBH}_{4(\mathrm{l})}+\mathrm{MgH}_{2(\mathrm{~s})} \rightarrow 320{ }^{\circ} \mathrm{C} \rightarrow \mathrm{Mg}_{(\mathrm{s})}+2 \mathrm{LiBH}_{4(\mathrm{l})}+\mathrm{H}_{2(\mathrm{~g})} \rightarrow 380{ }^{\circ} \mathrm{C} \rightarrow 2 \mathrm{LiH}_{(\mathrm{s})}+\mathrm{MgB}_{2(\mathrm{~s})}+4 \mathrm{H}_{2(\mathrm{~g})}
$$

Teniendo en cuenta la marcada mejora que se logró mediante el agregado del aditivo $\mathrm{Li}_{\mathrm{x}} \mathrm{TiO}_{2}$, se ha evaluado la reversibilidad y estabilidad a los sucesivos ciclos de absorción desorción de hidrógeno del material $2 \mathrm{LBHMH} 5 \mathrm{Li}_{\mathrm{x}} \mathrm{TiO}_{2}$. En la fig. $2 \mathrm{~A}$ y B se muestra la reversibilidad del material a $425^{\circ} \mathrm{C}$ luego de la primera desorción no-isotérmica (fig. 1 (b)). Se observa que el material es completamente reversible y sólo toma aproximadamente 15 minutos para la absorción-liberación de hidrógeno. Es de notar que la capacidad de almacenamiento de hidrógeno se ve ligeramente reducida, lo cual se relaciona a la alta presión de vapor del Mg metálico a alta temperatura. Sin embargo, al ciclar el material 15 veces a $400{ }^{\circ} \mathrm{C}$ (fig. $2 \mathrm{C}$ y D), temperatura menos severa para el $\mathrm{Mg}$, se obtienen capacidades reversibles del $\sim 8 \% \mathrm{p} / \mathrm{p} \mathrm{H} \mathrm{H}_{2}$ en 15 minutos para la hidrogenación y 35 minutos para la deshidrogenación. Este comportamiento cinético y la notable estabilidad al ciclado del material $2 \mathrm{LBHMH} 5 \mathrm{Li}_{\mathrm{x}} \mathrm{TiO}_{2}$ representan un importante avance respecto del material sin aditivo, 2LBHMH, con el cual se requieren tiempos de hidruración y deshidruración de $\sim 5$ horas y $\sim 12$ horas, respectivamente, obteniéndose capacidades de almacenamiento de hidrógeno $8-9 \%$ p/p [9], no muy superiores a las que se logran con el material $2 \mathrm{LBHMH} 5 \mathrm{Li}_{\mathrm{x}} \mathrm{TiO}_{2}$.
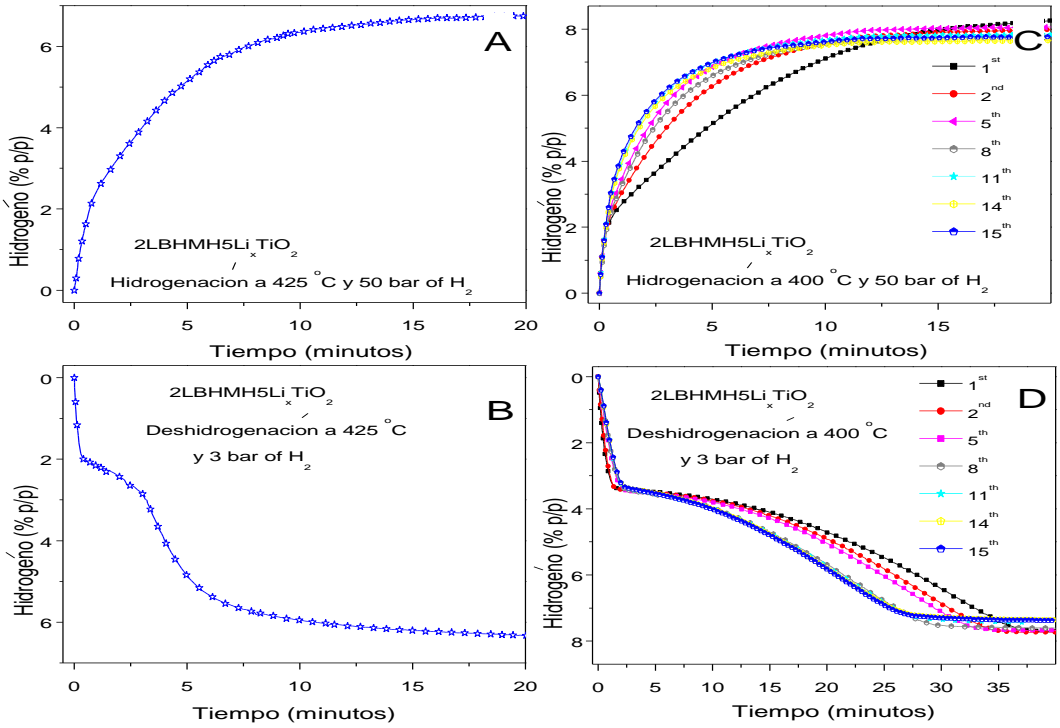

Figura 2: Comportamiento cinético y de estabilidad al ciclado para la hidrogenación-deshidrogenación isotérmicas para el material $2 \mathrm{LBHMH} 5 \mathrm{Li}_{\mathrm{x}} \mathrm{TiO}_{2}$ : A y B $-425^{\circ} \mathrm{C}, 3$ bar y 50 bar de $\mathrm{H}_{2}$. C y D $-400{ }^{\circ} \mathrm{C}, 3$ bar y 50 bar de $\mathrm{H}_{2}$ [15].

\subsection{Caracterización de los materiales luego del proceso de preparación por molienda mecánica y la interacción con hidrógeno}

En la figura 3 A y B se muestran los difractogramas de los materiales luego de la MM y de la primera deshidrogenación no-isotérmica (fig. 1). En el difractograma correspondiente al material 2LBHMH (fig. 3(c)) se observan solo los picos correspondientes al $\mathrm{MgH}_{2}$ y al $\mathrm{LiBH}_{4}$; producto de únicamente una mezcla física entre ellos luego de la molienda mecánica. Para el material 2LBHMH5TiB (fig. $3 \mathrm{~A}(\mathrm{a})$ ) se observa también la presencia de las fases $\mathrm{MgH}_{2}$ y $\mathrm{LiBH}_{4}$ junto con la del aditivo $\mathrm{TiB}_{2}$. En el caso del y $2 \mathrm{LBHMH}_{5} \mathrm{Li}_{\mathrm{x}} \mathrm{TiO}_{2}$ (fig. $3 \mathrm{~A}(\mathrm{c})$ ), es importante notar que se identifica la presencia de las mezcla de fases titanato $\mathrm{Li}_{\mathrm{x}} \mathrm{TiO}_{2}$ cuyos 
picos pertenecerían a las fases $\mathrm{Li}_{0,59} \mathrm{TiO}_{2}$ y $\mathrm{LiTiO}_{2}$; muchos de los picos de DRX se encuentran solapados con las fases hidruros mayoritarias en el materia. La molienda mecánica provee la energía necesaria para la interacción del $\mathrm{LiBH}_{4}$ y el TiO 2 que resulta en las fases $\mathrm{Li}_{\mathrm{x}} \mathrm{TiO}_{2}(\mathrm{x}=0,59$ y 1). Luego de la MM se verificó mediante el empleo de un detector de gases combustibles Bacharach que dentro de la cámara de molienda existía la presencia de hidrógeno gaseoso. Por lo tanto, en acuerdo con los resultados de DRX (fig. 3 A), el hidrógeno proviene de la descomposición del $\mathrm{LiBH}_{4}$.
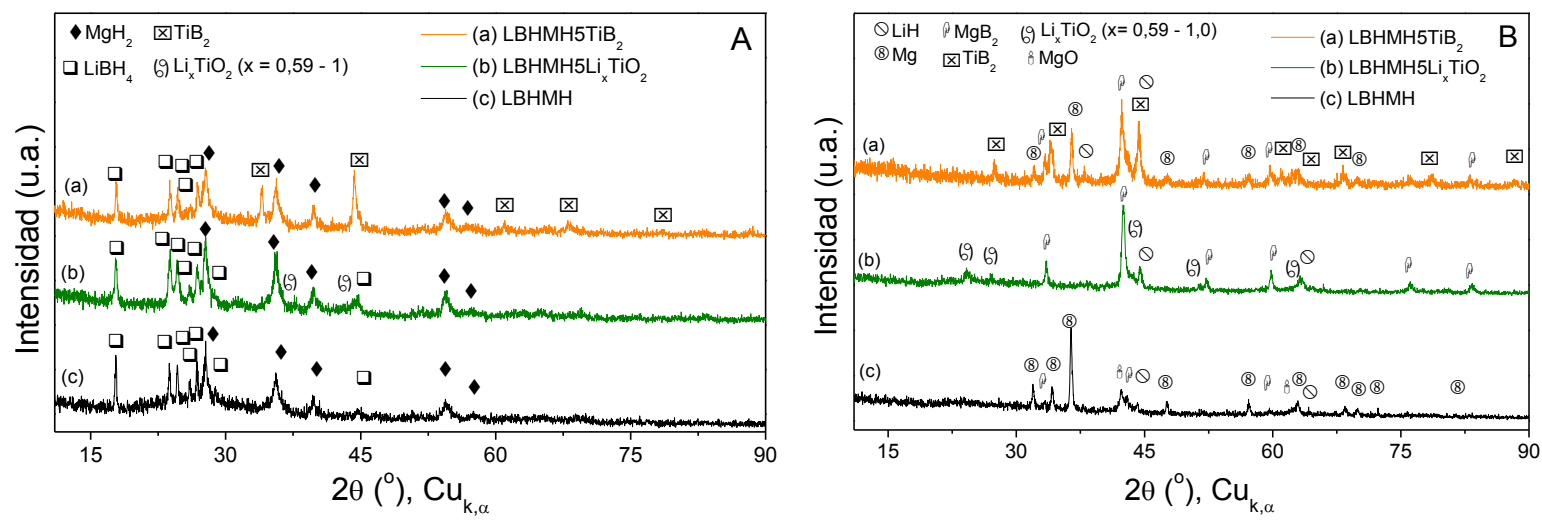

Figura 3: Caracterización por DRX: A - luego de la MM y B - Luego de la primera deshidrogenación para (a) 2LBHMH5TiB 2 , (b) 2LBHMH5 $\mathrm{Li}_{\mathrm{x}} \mathrm{TiO}_{2}$ y (c) $2 \mathrm{LBHMH}$.

Luego de la primera deshidrogenación no-isotérmica, para los materiales 2LBHMH y $2 \mathrm{LBHMHTiB}_{2}$ (fig. $3 \mathrm{~B}$ (c) y (b)) se detecta la presencia de $\mathrm{LiH}$ y de $\mathrm{MgB}_{2}$, ambos productos esperados de reacción. A su vez se aprecian reflexiones provenientes del $\mathrm{Mg}$ libre. Esto indica que la formación de la fase $\mathrm{MgB}_{2}$ no fue completa y confirma las bajas capacidades de hidrógeno obtenidas (fig. 1 (a) y (b)). En cambio, el difractograma del material $2 \mathrm{LBHMHLi}_{\mathrm{x}} \mathrm{TiO}_{2}$ (fig. 3 (a)) no muestra la presencia de $\mathrm{Mg}$ libre y claramente se pueden apreciar las reflexiones correspondientes al $\mathrm{LiH} \mathrm{y} \mathrm{MgB}_{2}$. A su vez, se notan reflexiones de las fases titanato $\mathrm{Li}_{\mathrm{x}} \mathrm{TiO}_{2}(\mathrm{x}=0,59$ y 1$)$.

Para poder verificar la presencia de fases amorfas luego de la primera deshidrogenación no isotérmica, se caracterizó a los materiales mediante espectroscopía Raman (ER). En la fig. 4 se pueden apreciar los espectros. El rango energético se seleccionó para detectar la presencia de la fase amorfa y estable $\mathrm{L}_{2} \mathrm{~B}_{12} \mathrm{H}_{12} \mathrm{y}$ se empleo el trabajo de Kim y colaboradores para la identificación de las fases [7]. Se puede observar que tanto en las muestras $2 \mathrm{LBHMH}$ y $2 \mathrm{LBHMHTiB}_{2}$ hay presencia de la fase amorfa y estable $\mathrm{L}_{2} \mathrm{~B}_{12} \mathrm{H}_{12}$, cuya formación se corresponde con la lenta cinética observada (fig. $1 \mathrm{~A}$ ) y la obtención incompleta de $\mathrm{MgB}_{2}$. En cambio el material $2 \mathrm{LBHMH} 5 \mathrm{Li}_{\mathrm{x}} \mathrm{TiO}_{2}$ no presenta dicha fase amorfa. Los resultados obtenidos por medio de la caracterización por DRX y ER sugieren que la presencia de las fases titanato $\mathrm{Li}_{\mathrm{x}} \mathrm{TiO}_{2}$ facilitan la formación de la fase $\mathrm{MgB}_{2}$ y resulta en una notable mejora de la cinética de deshidrogenación del sistema hidruro dado que evita la formación del compuesto amorfo y estable $\mathrm{L}_{2} \mathrm{~B}_{12} \mathrm{H}_{12}$, y permite que la liberación de hidrógeno se lleve a cabo en dos etapas como se describe en la reacción (4). Adicionalmente, es importante destacar que en otros análisis de DRX del material $2 \mathrm{LBHMH}_{5} \mathrm{Li}_{\mathrm{x}} \mathrm{TiO}_{2}$ (no mostrados aquí) se vió que luego de la rehidrogenación y deshidrogenación a $425^{\circ} \mathrm{C}$ (Fig. $2 \mathrm{~A}$ y B) las fases hidruros $\left(\mathrm{LiBH}_{4}\right.$ y $\mathrm{MgH}$ ) se regeneran completamente y no se ha notado la presencia de $\mathrm{Mg}$ libre, lo cual confirma la reversibilidad del sistema hidruro. 


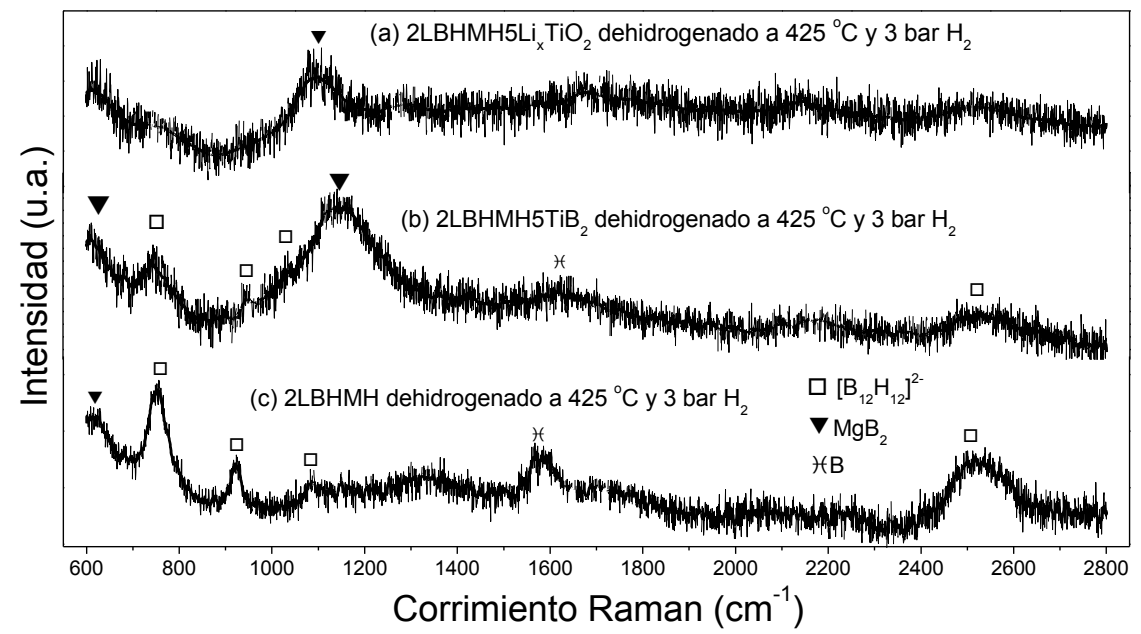

Figura 4: Caracterización los materiales por ER luego de la primera deshidrogenación no isotérmica (fig. 1A).

\subsection{Efecto de las fases titanato $\mathrm{Li}_{\mathrm{x}} \mathrm{TiO}_{2}$ sobre las propiedades cinéticas}

La fig. 5 muestra las imágenes de MET de alta resolución con sus respectivas Transformadas de Fourier (o FFT) y simulaciones de las estructuras cristalinas de las nanopartículas de $\mathrm{Li}_{\mathrm{x}} \mathrm{TiO}_{2}$ en el material 2LBHMH5 $\mathrm{Li}_{\mathrm{x}} \mathrm{TiO}_{2}$ luego de la $\mathrm{MM}$ y de la primera deshidrogenación no-isotérmica a $425{ }^{\circ} \mathrm{C}$ (fig. 5 A y B). También se pueden observar las distribuciones de tamaño de las partículas de $\mathrm{MgB}_{2}$ y fases titanto $\mathrm{Li}_{\mathrm{x}} \mathrm{TiO}_{2}$ luego de la primera deshidrogenación no-isotérmica (fig. $5 \mathrm{C}$ ). El análisis de las imágenes de MET del material 2 $\mathrm{LBHMH} \mathrm{Li}_{\mathrm{x}} \mathrm{TiO}_{2}$ luego de la $\mathrm{MM}$ y la deshidrogenación confirman la presencia de las fases titanato $\mathrm{Li}_{0.59} \mathrm{TiO}_{2}$ y $\mathrm{LiTiO}_{2}$, en acuerdo con los resultados obtenidos por DRX (fig. 3). Las nanopartículas de las fases $\mathrm{Li}_{\mathrm{x}} \mathrm{TiO}_{2}$ encontradas luego de la MM y deshidrogenación presentan tamaños de entre $10-15 \mathrm{~nm}$ (fig. 5 A (a), B (a) y (b)). A su vez, es de notar que luego de la MM se han observado nanopartículas de LiTiO 2 del orden de 3-4 nm (fig. 5 A (b)). Luego de la deshidrogenación (fig. 5 C (c) y (d)) la distribución de tamaños muestra que también existen nanopartículas de $\mathrm{Li}_{\mathrm{x}} \mathrm{TiO}_{2}$ de entre $1-6 \mathrm{~nm}$. Se ha verificado que la distribución de las fases $\mathrm{Li}_{\mathrm{x}} \mathrm{TiO}_{2}$ en el material $2 \mathrm{LBHMH} 5 \mathrm{Li}_{\mathrm{x}} \mathrm{TiO}_{2}$ es homogénea y dada la naturaleza del tamaño nanométrico de estas fases, su presencia evita la aglomeración de las fases hidruro y ello contribuye a la mejora su propiedades cinéticas [5].
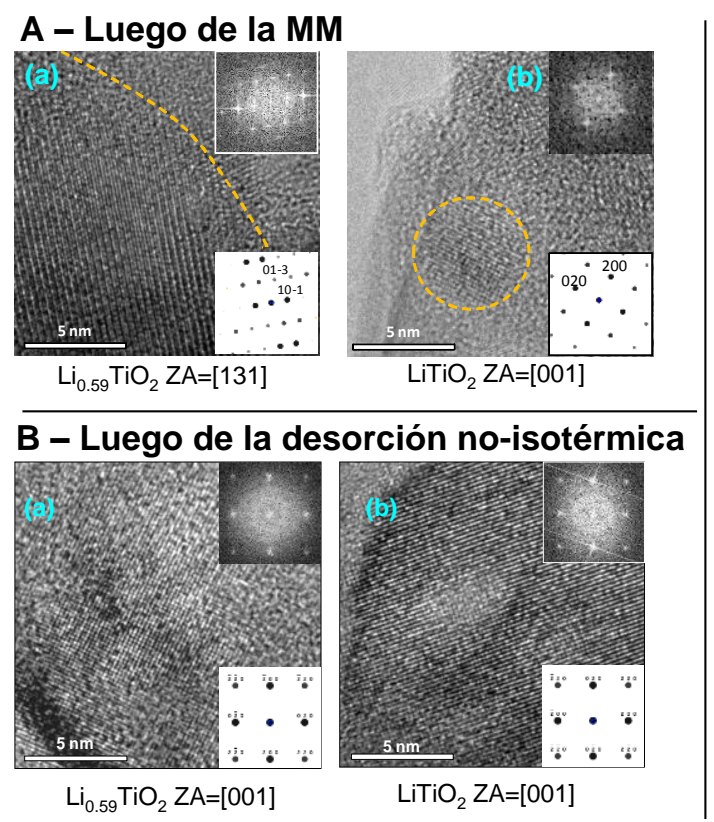

\section{C - Distribución de tamaños luego de la desorción no-isotérmica}
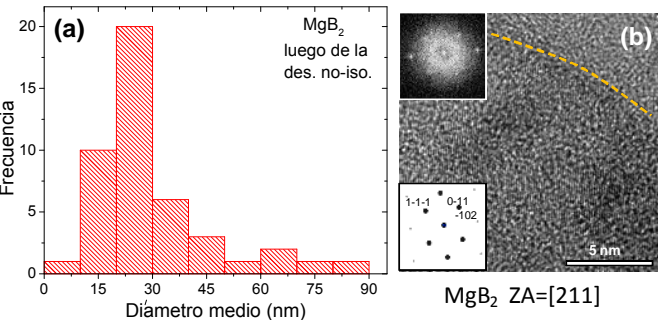

Tamaño medio: $30 \mathrm{~nm}$ / Rango: 10 - $60 \mathrm{~nm}$

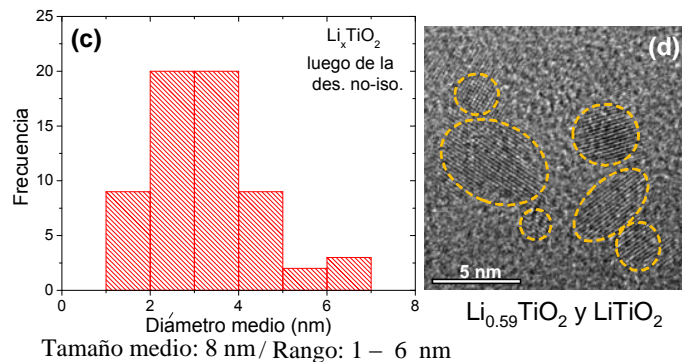

Figura 5: Imágenes de MET de alta resolución, Transformada de Fourier (esq. Superior derecha) y estructura cristalina simulada (esq. inferior derecha) de las nanopartículas de $\mathrm{Li}_{\mathrm{x}} \mathrm{TiO}_{2}$ del material $2 \mathrm{LBHMH}_{2} \mathrm{Li}_{\mathrm{x}} \mathrm{TiO}_{2}$ : A - luego de la $\mathrm{MM}$ y B - Luego de la primera deshidrogenación no-isotérmica y C - Distribución de tamaños luego de la primera deshidrogenación no-isotérmica para las partículas de $\mathrm{Li}_{\mathrm{x}} \mathrm{TiO}_{2}$ y $\mathrm{MgB}_{2}$. 
El $\mathrm{MgB}_{2}$ luego de la deshidrogenación, fase que hace posible que el sistema hidruro sea reversible, presenta una distribución de tamaños de entre $10-60 \mathrm{~nm}$ (fig. $5 \mathrm{C}$ (a)). El cálculo del tamaño de grano de $\mathrm{MgB}_{2}$ a partir del difractograma del material 2LBHMH5Li $\mathrm{LiO}_{2}$ (fig. $3 \mathrm{~B}$ (b)) arroja un valor de $25 \mathrm{~nm}$, lo cual se encuentra dentro del rango de distribuciones de tamaños obtenido por medio del MET y muy cercano al tamaño medio de $30 \mathrm{~nm}$. Por lo tanto, el reducido tamaño del $\mathrm{MgB}_{2}$ indica que la presencia de las nanopartículas de $\mathrm{Li}_{\mathrm{x}} \mathrm{TiO}_{2}$ favorece la retención de tamaños de fase mayoritarias en rangos nanométricos, facilitando la difusión de las especies y contribuyendo también a la mejora del comportamiento cinético observado.

En la fig. 6 se muestran las curvas de CDB-AP y TG para los materiales 2LBHMH y 2LBHMH5Li $\mathrm{LiO}_{2}$. Para el caso del material 2LBHMH (fig. 6 (a), la curva de CDB-AP muestra un primer evento térmico con un máximo a $113{ }^{\circ} \mathrm{C}$ que corresponde a la transición de fase reversible del $\mathrm{LiBH}_{4} \mathrm{desde}^{\circ}$ un sistema ortorrómbico a hexagonal, reportada entre $105-120^{\circ} \mathrm{C}$. Luego, con un máximo de $288^{\circ} \mathrm{C}$ se observa un segundo evento térmico que corresponde a la fusión del $\mathrm{LiBH}_{4}$, reportado entre $260-290{ }^{\circ} \mathrm{C}$ $[4,10]$. Finalmente, el máximo del tercer evento térmico se encuentra a $385^{\circ} \mathrm{C}$ y pertenece a la descomposición del $\mathrm{MgH}_{2}$ [4]. Mediante la CDB-AP no es posible caracterizar la descomposición del $\mathrm{LiBH}_{4}$ y subsecuente formación del $\mathrm{MgB}_{2}$ dado que, como se ha mostrado en la fig. 1 (a), en la primera y segunda etapa (reacciones (2) y (3), sección 3.1) son muy lentas. En la curva correspondiente a la técnica de TG para el material 2LBHMH (fig. 6 (a)), no se nota pérdida de masa asociada a la liberación de hidrógeno hasta los aproximadamente $350^{\circ} \mathrm{C}$, lo que esta en acuerdo al evento endotérmico correspondiente a la descomposición del $\mathrm{MgH}_{2}$.

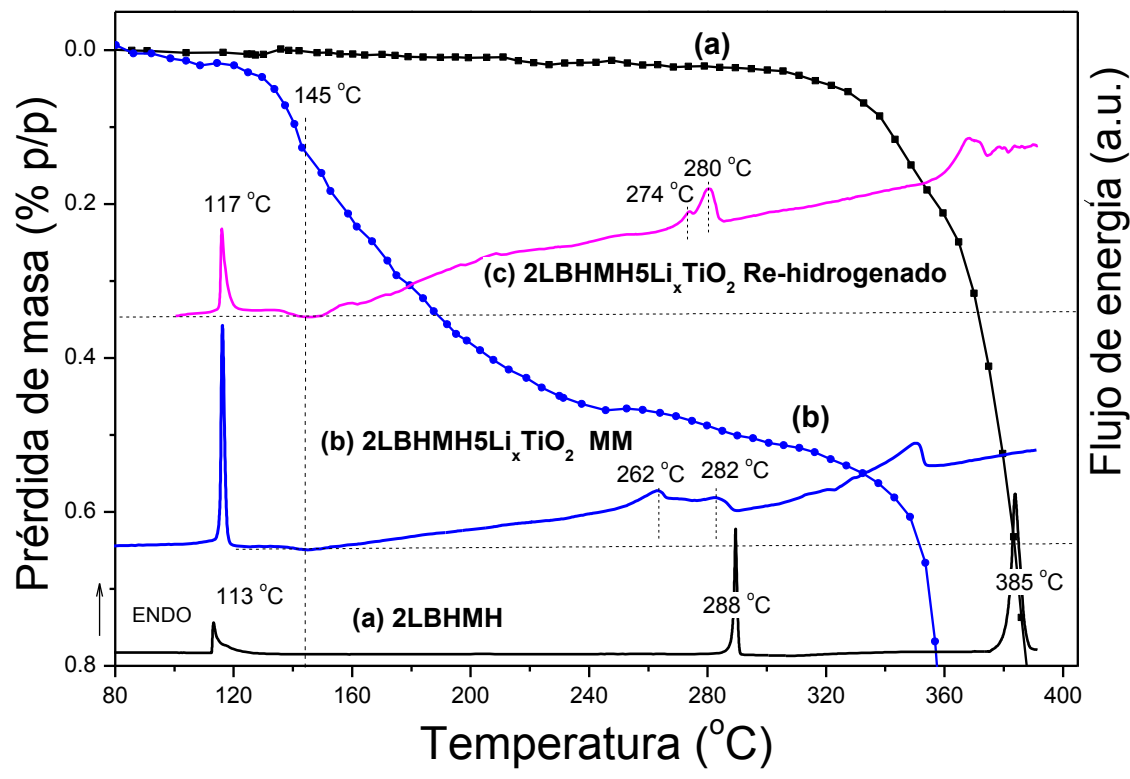

Figura 6: Análisis térmico para los materiales $2 \mathrm{LBHMH}$ y $2 \mathrm{LBHMH}^{2} \mathrm{Li}_{\mathrm{x}} \mathrm{TiO}_{2}$. A - TG y B - TG y HP-DSC.

Para el caso del material 2LBHMH5Li $\mathrm{TiO}_{2}$ luego de la MM y re-hidrogenación (fig. 6 (b) y (c)) se nota un comportamiento muy distinto al observado para el material 2LBHMH (fig. 6 (a)). El primer evento térmico para el material $2 \mathrm{LBHMH} 5 \mathrm{Li}_{\mathrm{x}} \mathrm{TiO}_{2}$ posee un máximo a $117^{\circ} \mathrm{C}$, lo cual se encuentra dentro del rango de temperaturas reportado [4,10]. Por lo tanto, la transición de fase del $\mathrm{LiBH}_{4}$ no se ve modificada por la presencia de $\mathrm{Li}_{\mathrm{x}} \mathrm{TiO}_{2}$. A $150{ }^{\circ} \mathrm{C}$ aproximadamente, el material $2 \mathrm{LBHMH} \mathrm{Li}_{\mathrm{x}} \mathrm{TiO}_{2}$ luego de la $\mathrm{MM}$ y rehidrogenación presenta una notable desviación respecto de la línea base y posee entre $\sim 260{ }^{\circ} \mathrm{C}$ a $280{ }^{\circ} \mathrm{C}$ eventos térmicos solapados. Los eventos térmicos a temperaturas por encima de los $300^{\circ} \mathrm{C}$ se corresponden con la descomposición de las fases hidruro (fig. 6 (b) y (c)). Esto sugiere que a partir de $150{ }^{\circ} \mathrm{C}$ y durante la transición de fase del $\mathrm{LiBH}_{4}$ el aditivo $\mathrm{Li}_{\mathrm{x}} \mathrm{TiO}_{2}$ interactúa con el $\mathrm{LiBH}_{4}$. Por medio de la técnica de $\mathrm{TG}$ se observa que el material luego de la molienda mecánica (fig. 6 (b)) comienza a perder masa alrededor de los $120^{\circ} \mathrm{C}$. Esta pérdida continúa hasta los $\sim 320^{\circ} \mathrm{C}$, temperatura a la cual se nota un cambio de pendiente abrupto y que se corresponde con el comienzo de la descomposición del $\mathrm{MgH}_{2}$ (fig. 6 (b)), en concordancia con la fig. 1 (c). El material $2 \mathrm{LBHMH}_{5} \mathrm{Li}_{\mathrm{x}} \mathrm{TiO}_{2}$ luego de la $\mathrm{MM}$ se calentó hasta $200{ }^{\circ} \mathrm{C}$ en un recipiente hermético y luego mediante el empleo del detector de gases combustibles Bacharach se verificó la existencia 
de hidrógeno. Esto verifica que tanto los procesos observados en las curvas de CDB-AP (fig. 6 (b) y (c)) como la pérdida de masa medida en la curva de TG (fig. 6 (b)) se relacionan con la liberación de hidrógeno.

En trabajos previos se ha encontrado que la interacción entre el $\mathrm{LiBH}_{4}$ y $\mathrm{TiO}_{2}$ resulta en la formación de la fase $\mathrm{LiTiO}_{2}$. Esta reacción libera solamente hidrógeno en fase gaseosa y comienza a ocurrir entre $100{ }^{\circ} \mathrm{C}$ y $150{ }^{\circ} \mathrm{C}$, presentando un máximo de liberación de hidrógeno entre $275^{\circ} \mathrm{C}$ y $290^{\circ} \mathrm{C}[11,12]$. También, las nanopartículas de $\mathrm{Li}_{x} \mathrm{TiO}_{2}$ se conocen como especies polimórficas donde el $\mathrm{Li}^{+}$se encuentra intercalado dentro de la red del $\mathrm{TiO}_{2}$. El Li ${ }^{+}$posee una alta movilidad a través de la red de $\mathrm{Li}_{\mathrm{x}} \mathrm{TiO}_{2}$. Los procesos difusivos del $\mathrm{Li}^{+}$, los cuales se incrementan a mayores temperaturas, hacen posible que el titanato $\mathrm{Li}_{0.59} \mathrm{TiO}_{2}$ pueda saturarse obteniéndose la especie $\mathrm{LiTiO}_{2}[13,14]$. En acuerdo con la bibliografía [11-14] y a lo que se observa en las curvas de CDB-AP y TG para el material $2 \mathrm{LBHMH}_{5} \mathrm{Li}_{\mathrm{x}} \mathrm{TiO}_{2}$, se propone que la fase titanato $\mathrm{Li}_{\mathrm{x}} \mathrm{TiO}_{2}$ interactúa con la fase hidruro $\mathrm{LiBH}_{4}$ mediante la siguiente reacción (5):

$0,41 \mathrm{LiBH}_{4(\mathrm{~s}-\mathrm{l})}+\mathrm{Li}_{0,59} \mathrm{TiO}_{2(\mathrm{~s})} \rightarrow \mathrm{LiTiO}_{2(\mathrm{~s})}+0,41 \mathrm{~B}_{(\mathrm{s})}+0,82 \mathrm{H}_{2(\mathrm{~g})}$

Los resultados obtenidos permiten decir que el efecto del aditivo se relaciona con la descomposición temprana del $\mathrm{LiBH}_{4}$ mediante la reacción con la fase titanato menos saturada en litio $\left(\mathrm{Li}_{0,59} \mathrm{TiO}_{2}\right)$ y la subsecuente obtención de la fase titanato saturada $\left(\mathrm{LiTiO}_{2}\right)$, en acuerdo con los recientes resultados obtenidos por Puszkiel et al. [15]. Ello posibilita disponer de $\mathrm{B}_{(\mathrm{s})}$ libre para la formación de núcleos de $\mathrm{MgB}_{2}$ una vez que se tiene cierta cantidad de $\mathrm{Mg}_{(\mathrm{s})}$. En concordancia con el mecanismo propuesto por Bösenberg et al. [8], la formación de núcleos de $\mathrm{MgB}_{2}$ permite que el resto de la fase se nuclee y crezca sobre sí misma. Mediante este mecanismo se suprime la formación de $\mathrm{Li}_{2} \mathrm{~B}_{12} \mathrm{H}_{12}$, según la reacción (4). Con base a los resultados obtenidos de la reversibilidad y ciclado del sistema (fig. 2), se infiere que el mecanismo de acción de las nanopartículas de $\mathrm{Li}_{x} \mathrm{TiO}_{2}$ es reversible.

\section{CONCLUSIONES}

La adición de nanopartículas de titanatos $\mathrm{Li}_{\mathrm{x}} \mathrm{TiO}_{2}$ al sistema hidruro $2 \mathrm{LiBH}_{4}+\mathrm{MgH}_{2}$ mejora notablemente el comportamiento cinético, reduciendo los tiempos de liberación de hidrógeno para la primera deshidrogenación desde $35 \mathrm{~h}$. a menos de $2 \mathrm{~h}$. A su vez, permiten que el sistema absorba y libere hidrógeno reversiblemente en tiempos apreciablemente más cortos. La notable mejora se debe a que la presencia de fases titanato $\left(\mathrm{Li}_{\mathrm{x}} \mathrm{TiO}_{2}, \mathrm{x}=0,59\right.$ y 1) promueve la descomposición temprana de $\mathrm{LiBH}_{4}$ evitando la formación de la fase amorfa y estable $\mathrm{Li}_{2} \mathrm{~B}_{12} \mathrm{H}_{12}$, paso intermedio que resulta ser extremadamente lento y que hace que la formación del $\mathrm{MgB}_{2}$ durante la deshidrogenación no sea completa. A su vez la presencia de nanopartículas de titanatos $\mathrm{Li}_{\mathrm{x}} \mathrm{TiO}_{2}$ evita la aglomeración de las fases hidruro del material $2 \mathrm{LiBH}_{4}+\mathrm{MgH}_{2}$, favorece a la retención de tamaños nanométricos de la fase $\mathrm{MgB}_{2}$ y mejora los procesos difusivos del $\mathrm{Li}^{+}$. Estos efectos contribuyen a la notable mejora observada en el comportamiento cinético.

\section{AGRADECIMIENTOS}

Los autores agradecen al CONICET (Consejo Nacional de Investigaciones Científicas y Técnicas), ANPCyT (Agencia Nacional de Promoción Científica y Tecnológica) - PICT-2015-1865, CNEA (Comisión Nacional de Energía Atómica) por el apoyo financiero para llevar a cabo el presente trabajo. A la División Física de Metales del $\mathrm{CAB}$ por el acceso a las instalaciones del microscopio de transmisión, a la División de Caracterización de Materiales por el acceso al equipo de difracción de rayos X. A su vez, se agradece al comité organizador del congreso SAM-CONAMET-2016 por el apoyo financiero para la asistencia al congreso del estudiante de grado Hernán Martinelli.

\section{BIBLIOGRAFÍA}

[1] ZÜTTEL, A., "Materials for hydrogen storage"; Materials Today, v. 6, n. 9, pp. 24-33, Sept. 2003.

[2] VAJO, J.J., SKEITH, S.L., MERTENS, F., "Reversible storage of hydrogen in destabilized $\mathrm{LiBH}_{4}$ ". J. Phys. Chem. B, v 109, n. 9, pp. 3719-3722, Diciembre 2005.

[3] BARKOHRDARIAN, G., KLASSEN, T., DORNHEIM, M., et al., "Unexpected kinetic effect of $\mathrm{MgB}_{2}$ in reactive hydride composites containing complex borohydrides", J. Alloys Compd., v. 440, pp. L18-L21, Noviembre 2007.

[4] BÖSENBERG, U., DOPPIU, S., MOSEGAARD, L., et al., "Hydrogen sorption properties of $\mathrm{MgH}_{2}-$ $\mathrm{LiBH}_{4}$ composites", Acta Mater. , v. 55, n. 8, pp. 3951-3958, Marzo 2007. 
[5] PUSZKIEL, J., CASTRO RIGLOS, M.V., RAMALLO-LÓPEZ, J., et al., "Efecto de la formación in-situ de nanopartículas con Ti sobre el $2 \mathrm{LiH}-\mathrm{MgB}_{2}$ para almacenamiento de hidrógeno", In: Anales del congreso SAM/CONCAMET 2015, pp. 1-6, Concepción - Chile, Noviembre 2015.

[6] ALEXANDER, L., KLUG, P.H., "Determination of cristallite size with the x-ray spectrometer", J. Appl. Phys., v. 21, n 2, pp. 137 - 147, Julio 1950.

[7] KIM, K-B., SHIM, J-H., PARK, S-H., et al., "Dehydrogenation reaction pathway of the $\mathrm{LiBH}_{4}-\mathrm{MgH}_{2}$ composite under various pressure conditions", J. Phys. Chem. C, v. 119, n. 18, pp. 9714-9720, Abril 2015.

[8] BÖSENBERG, U., KIM, J.W., GOSSLAR, D., et al., "Role of additives in $\mathrm{LiBH}_{4}-\mathrm{MgH}_{2}$ reactive hydride composites for sorption kinetics", Acta mater., v. 58, n. 9, pp. 3381-3389, Mayo 2010.

[9] PUSZKIEL, J.A., GENNARI, F.C., ARNEODO LAROCHETTE, P., et al., "Effect of Fe additive on the hydrogenation-dehydrogenation properties of $2 \mathrm{LiBH}_{4}+\mathrm{MgB}_{2} / \mathrm{LiBH}_{4}+\mathrm{MgH}_{2}$ system". J. of Power Sources, v. 284, n. 15, pp. 606-616, Junio 2015.

[10] NAKAGAWA, T., ICHIKAWA, T., HANADA, H., et al., "Thermal analysis on the Li-Mg-B-H systems", J. Alloys Compd., v. 446-447, n. 31, pp. 306-309, Octubre 2007.

[11] YU, X.B., GRANT, D.M., WALKER, G.S., "Low-temperature dehydrogenation of $\mathrm{LiBH}_{4}$ through destabilization with $\mathrm{TiO}_{2}$ ”, J. Phys. Chem. C, v. 112, n. 29, pp. 11059-11062, Julio 2008.

[12] LIU, H., JIAO, L., ZHAO, Y., et al., "Improved dehydrogenation performance of $\mathrm{LiBH}_{4}$ by confinement into porous $\mathrm{TiO}_{2}$ micro-tubes", J. Mater. Chem. A, v. 2, n. 24, pp. 9244-9250, Abril 2014.

[13] YANG, Z., CHOI, D., KERISIT, S., et al., "Nanostructures and lithium electrochemical reactivity of lithium titanites and titanium oxides: A review", J. of Power Sources, v. 192, n. 2, pp. 588-598, Julio 2009.

[14] BORGHOLS, W.J.H., LÜTZENKIRCHEN-HECHT, D., HAAKE, U., et al., "The electronic structure and ionic diffusion of nanoscale $\mathrm{LiTiO}_{2}$ anatase", Phys. Chem. Chem. Phys., v. 11, n. 27, pp. 5742-5748, Mayo 2009.

[15] PUSZKIEL, J.A., CASTRO RIGLOS, M.V., KARIMI, F., et al., "Changing the dehydrogenation pathway of $\mathrm{LiBH}_{4}-\mathrm{MgH}_{2}$ via nanosized lithiated $\mathrm{TiO}_{2}$ ", Phys.Chem.Chem.Phys., v. 11, n. 19, pp. 74557460, Enero 2017. 